\title{
Dispersion relations in ultradegenerate relativistic plasmas
}

\author{
Cristina Manuel* \\ Theory Division, CERN, CH-1211 Geneva 23, Switzerland \\ (Received 15 May 2000; published 12 September 2000)
}

\begin{abstract}
The propagation of excitation modes in a relativistic ultradegenerate plasma is modified by their interactions with the medium. These modifications can be computed by evaluating their on-shell self-energy, which gives (gauge-independent) dispersion relations. For modes with momentum close to the Fermi momentum, the one-loop fermion self-energy is dominated by a diagram with a soft photon in the loop. We find the one-loop dispersion relations for quasiparticles and antiquasiparticles, which behave differently as a consequence of their very different phase-space restrictions when they scatter with the electrons of the Fermi sea. In a relativistic system, the unscreened magnetic interactions spoil the normal Fermi-liquid behavior of the plasma. For small values of the Fermi velocity, we recover the nonrelativistic dispersion relations of condensed-matter systems.
\end{abstract}

PACS number(s): 11.10.Wx, 12.20.Ds, 12.38.Mh, 52.60.+h

\section{INTRODUCTION}

The study of hot relativistic plasmas is nowadays a very active field of research [1]. This is due to the existence of experimental programs to test the existence of the quarkgluon plasma phase of QCD. The physics of some astrophysical settings, such as those of neutron stars and supernovas, also requires knowledge of a different regime of relativistic plasmas, less hot but still very dense. This cold and ultradegenerate regime of QED and QCD has been much less explored. However, the fact that matter at very high baryonic densities behaves as a color superconductor has given us a strong motivation to study ultradegenerate relativistic plasmas, as several new phenomena occur in this phase of QCD (see [2] and [3], and references therein).

One of the central concepts in a plasma is that of a quasiparticle. A particle immersed in a medium modifies its propagation properties by interacting with the surrounding medium. In field-theoretical language, we would say that the particle is "dressed" by a self-energy cloud. In the ultradegenerate plasma the relevant degrees of freedom are those of quasiparticles or quasiholes (absences of particles in the Fermi sea) living close to the Fermi surface. Because of the exclusion principle, quasiparticles or quasiholes can only live if they are outside or inside the Fermi sea, respectively. These excitations tend to lower their energy by undergoing collisions with the particles in the Fermi sea. They decay, and thus have a finite lifetime. The concept of quasiparticle, however, only makes sense if its lifetime is long enough, or in other words, if its damping rate is much smaller than its energy.

Here we will mainly be concerned with electromagnetic plasmas. There is a vast literature on the quasiparticle properties in nonrelativistic cold plasmas [4]. The same does not hold true for the relativistic ones, though. There are two main differences in these two energy regimes of a plasma. In the nonrelativistic domain, the electric interactions are dominant, while the magnetic ones are suppressed by a factor

*Email address: Cristina.Manuel@cern.ch $(v / c)^{2}$, where $v$ is the velocity of the particle, and $c$ is the velocity of light. Thus, magnetic interactions start to be relevant only when quasiparticles are fast enough or, in other words, when the Fermi velocity $v_{F}$ approaches the velocity of light. This is an important difference, as electric interactions are not long ranged in the medium, because of Debye screening, while magnetic interactions are. The relevance of this last point has already been stressed in the condensedmatter literature [5], just noticing that magnetic interactions spoil the normal Fermi-liquid behavior of the plasma. The second main difference is due to the fact that in a relativistic plasma there are also antiparticle excitations. Their contribution to any physical process is in general suppressed, since it takes more energy to excite an antiparticle than a particle of the Fermi-Dirac sea. Nevertheless, in the context of the color flavor-locking phase of QCD [6], some of the properties of the antiparticles determine the mass spectrum of the Goldstone modes which arise from the spontaneous breaking of chiral symmetry, so those cannot be neglected.

In this paper we study the quasiparticle and antiquasiparticle dispersion relations in a full relativistic framework, generalizing the results of a previous publication [9] to the case where the Fermi velocity $v_{F} \neq c$. We can thus explore all the energy domains of the system, and in particular, we can take the nonrelativistic limit $v_{F} \ll c$, and match the results obtained in the condensed-matter literature $[4,5]$. The dispersion relations are obtained by computing the on-shell oneloop self-energy. While the one-loop self-energy is in general gauge dependent, it is not when evaluated on the particles mass shell. For quasiparticles with momenta close to the Fermi momentum, the one-loop self-energy is dominated by a diagram in which the photon is soft. When the photon is soft, it also needs to be dressed to take properly into account the effects of the medium. This can be done by using the resummation techniques proposed by Braaten and Pisarski [7], and considering hard thermal loop photon propagators, or hard dense loop (HDL) ones for the ultradegenerate case [8]. We first compute the on-shell imaginary part of the one-loop self-energy for electrons and positrons, which can be interpreted in terms of their scattering with 
particles of the Fermi sea, via an exchange of a soft photon. The on-shell real part of the self-energy can be reconstructed from the on-shell imaginary part, just by using a KramersKroning relation.

This paper is structured as follows. Section II introduces the notation of the paper. We work in natural units, $c=\hbar$ $=k_{B}=1$, unless otherwise stated. In Sec. III A we compute the on-shell one-loop self-energy of the fermion. We take the nonrelativistic limit of our results in Sec. III B, and conclude in Sec. IV. In Appendix A the spectral functions of the HDL photon propagators are given for $v_{F} \neq c$, and in Appendix B Luttinger's theorem is recalled.

\section{DISPERSION RELATIONS FOR THE QUASIPARTICLES}

We consider a plasma with a finite density of electrons, characterized by a chemical potential $\mu$. In order to guarantee its stability, we assume that the electrons are immersed in a uniform background of positive charges, of density equal to the average electron density. These background charges can be due to positively charged ions, which are very heavy.

In a plasma with chemical potential $\mu$, the propagation properties of the quasiparticles are modified by medium effects. The dressed fermion propagator $S(P)$, where $P$ $=\left(p_{0}, \mathbf{p}\right)$ is the four momentum, obeys the SchwingerDyson equation

$$
S^{-1}(P)=S_{0}^{-1}(P)+\Sigma(P)
$$

where $S_{0}^{-1}$ is the inverse free propagator

$$
S_{0}^{-1}(P)=\not P+\mu \gamma_{0}-m,
$$

with $\not P=P^{\mu} \gamma_{\mu}$ and $\Sigma(P)$ is the one-loop self-energy.

Because of the clear asymmetry between electrons and positrons in the electromagnetic plasma, it is convenient to treat them separately, as their propagation properties will be modified in different ways. Introducing the positive and negative energy projectors

$$
\Lambda_{\mathbf{p}}^{ \pm}=\frac{E_{p} \pm\left(\gamma_{0} \gamma \cdot \mathbf{p}+m \gamma_{0}\right)}{2 E_{p}},
$$

where $E_{p}=\sqrt{p^{2}+m^{2}}$, we can rewrite

$$
\begin{gathered}
S_{0}^{-1}(P)=\gamma_{0} \Lambda_{\mathbf{p}}^{+}\left(p_{0}+\mu-E_{p}\right)+\gamma_{0} \Lambda_{\mathbf{p}}^{-}\left(p_{0}+\mu+E_{p}\right), \\
\Sigma(P)=\gamma_{0} \Lambda_{\mathbf{p}}^{+} \Sigma_{+}(P)-\gamma_{0} \Lambda_{\mathbf{p}}^{-} \Sigma_{-}(P) .
\end{gathered}
$$

After inverting Eq. (1) one gets

$$
S(P)=S_{+}(P) \Lambda_{\mathbf{p}}^{+} \gamma_{0}+S_{-}(P) \Lambda_{\mathbf{p}}^{-} \gamma_{0},
$$

where

$$
S_{ \pm}(P)=\frac{1}{p_{0}+\mu \mp\left[E_{p}-\Sigma_{ \pm}(P)\right]},
$$

and the upper or lower subscripts refer to electrons or positrons, respectively.

Every energy eigenstate can be projected onto states of given helicity, with the projectors

$$
\mathcal{P}^{ \pm}(\mathbf{p})=\frac{1 \pm \gamma_{5} \gamma_{0} \gamma \cdot \hat{\mathbf{p}}}{2}
$$

In principle, the most general structure of the one-loop selfenergy contains four unknown functions, according to the energies and helicities of the quasiparticles. However, the effects which will be discussed in this paper do not depend on the helicity of the quasiparticles, and thus we would not explicitly take into account the helicity projectors.

The value of the one-loop self-energy $\Sigma$ is gauge dependent. However, when it is evaluated on the particles mass shell, it should be gauge independent. This is so because the poles of Eq. (7) give the physical dispersion relations of electrons and positrons which define their propagation properties in the plasma.

The dispersion relations obtained from Eq. (7) are

$$
\begin{aligned}
& \omega_{ \pm}=-\mu \pm\left[E_{p}-\operatorname{Re} \Sigma_{ \pm}\left(\omega_{ \pm}+i \gamma_{ \pm}, \mathbf{p}\right)\right], \\
& \gamma_{ \pm}=\mp \operatorname{Im} \Sigma_{ \pm}\left(\omega_{ \pm}+i \gamma_{ \pm}, \mathbf{p}\right),
\end{aligned}
$$

where $\omega_{ \pm}$and $\gamma_{ \pm}$define the energy and damping rates of the electrons or positrons, respectively. For the concept of quasiparticle to make sense, it is necessary that $\gamma_{ \pm} \ll \omega_{ \pm}$, so that the quasiparticles are long lived enough.

In the remaining part of the paper the dispersion relations for quasiparticles and antiquasiparticles with momentum close to the Fermi momentum will be studied. In this case, the dominant contribution to their one-loop self-energy arises when the photon in the loop is soft, that is, of order $\sim e \mu$, where $e$ is the electromagnetic coupling constant. When the photon is soft it has also to be dressed, in order to take into account properly the medium effects of Debye screening and Landau damping.

\section{THE ON-SHELL FERMION SELF-ENERGY}

\section{A. Relativistic domain}

For a plasma at temperature $T$ and chemical potential $\mu$, we compute the one-loop self-energy $\Sigma$ using the imaginary time formalism. It is convenient to use the spectral function representation of the fermion and photon propagators in the computation. The free fermion propagator is given by

$$
S_{0}\left(i \omega_{n}, \mathbf{k}\right)=\int_{-\infty}^{\infty} \frac{d k_{0}}{2 \pi} \frac{(\mathbb{K}+m) \rho_{f}(K)}{k_{0}-i \omega_{n}-\mu},
$$

with

$$
\rho_{f}(K)=\frac{\pi}{E_{k}}\left[\delta\left(k_{0}-E_{k}\right)-\delta\left(k_{0}+E_{k}\right)\right] .
$$

In Eq. (11), $\omega_{n}=\pi(2 n+1) T$ is a fermionic Matsubara frequency. The (resummed) photon propagator $\Delta_{\mu \nu}(Q)$, where 
$Q=\left(i \omega_{s}, \mathbf{q}\right)$, and $\omega_{s}=2 \pi s T$ is a bosonic Matsubara frequency, is written in the Coulomb gauge

$$
\Delta_{\mu \nu}(Q)=\delta_{\mu 0} \delta_{\nu 0} \Delta_{L}(Q)+\mathcal{P}_{\mu \nu}^{T} \Delta_{T}(Q)+\xi_{C} \frac{Q_{\mu} Q_{\nu}}{q^{4}}
$$

where $\mathcal{P}_{i j}^{T}=\left(\delta_{i j}-\hat{q}_{i} \hat{q}_{j}\right), \quad \hat{q}^{i}=\mathbf{q}^{i} /|\mathbf{q}|, \quad \mathcal{P}_{i 0}^{T}=\mathcal{P}_{0 i}^{T}=\mathcal{P}_{00}^{T}=0$, and $\xi_{C}$ is the gauge parameter. The longitudinal and transverse propagators are written in terms of their spectral functions

$$
\begin{aligned}
& \Delta_{L}\left(i \omega_{s}, q\right)=\int_{-\infty}^{\infty} \frac{d q_{0}}{2 \pi} \frac{\rho_{L}\left(q_{0}, q\right)}{q_{0}-i \omega_{s}}-\frac{1}{q^{2}}, \\
& \Delta_{T}\left(i \omega_{s}, q\right)=\int_{-\infty}^{\infty} \frac{d q_{0}}{2 \pi} \frac{\rho_{T}\left(q_{0}, q\right)}{q_{0}-i \omega_{s}} .
\end{aligned}
$$

Analytical expressions for $\rho_{L, T}$ can be found in $[10,1]$ for the case of an ultrarelativistic $(m=0)$ plasma. At $T=0$, it is also possible to derive the spectral functions for $m \neq 0$ [8]. We present analytical expressions for the spectral functions in this case in Appendix A.

The one-loop self-energy

$$
\Sigma(P)=e^{2} T \sum_{s} \int \frac{d^{3} q}{(2 \pi)^{3}} \gamma_{\mu} S_{0}(P-Q) \gamma_{\nu} \Delta_{\mu \nu}(Q),
$$

when expressed in terms of the spectral functions, reads

$$
\begin{aligned}
\Sigma(i \omega, p)= & e^{2} T \sum_{n} \int \frac{d^{3} q}{(2 \pi)^{3}} \int_{-\infty}^{\infty} \frac{d k_{0}}{2 \pi} \rho_{f}(K) \gamma_{\mu}(\mathbb{K}+m) \gamma_{\nu} \\
& \times\left\{\left(\int_{-\infty}^{\infty} \frac{d q_{0}}{2 \pi} \frac{\delta_{\mu 0} \delta_{\nu 0} \rho_{L}\left(q_{0}, q\right)+\mathcal{P}_{\mu \nu}^{T} \rho_{T}\left(q_{0}, q\right)}{\left(q_{0}-i \omega_{n}\right)\left(k_{0}-i \omega+i \omega_{n}-\mu\right)}\right)\right. \\
& \left.-\frac{1}{q^{2}} \frac{\delta_{\mu 0} \delta_{\nu 0}}{\left(k_{0}-i \omega+i \omega_{n}-\mu\right)}+\xi_{C} \frac{Q_{\mu} Q_{\nu}}{q^{4}}\right\} .
\end{aligned}
$$

The sum over Matsubara frequencies is now easily performed. After analytical continuation $i \omega_{n}+\mu \rightarrow p_{0}+i \eta$, with $\eta \rightarrow 0^{+}$to Minkowski space, one can evaluate the onshell imaginary part. It is very easy to realize that none of the last two pieces of Eq. (16) contribute to this on-shell imaginary part. Therefore, the result of the computation is gauge independent. One finds

$$
\begin{aligned}
\operatorname{Im} \Sigma & \left(p_{0}+i \eta, p\right) \\
& =-e^{2} \operatorname{Im} \int \frac{d^{3} q}{(2 \pi)^{3}} \int_{-\infty}^{\infty} \frac{d k_{0}}{2 \pi} \rho_{f}(K) \\
& \times \int_{-\infty}^{\infty} \frac{d q_{0}}{2 \pi} \frac{1+f\left(q_{0}\right)-\widetilde{f}\left(k_{0}-\mu\right)}{p_{0}-k_{0}-q_{0}+i \eta} \\
& \times \gamma_{\mu}(\mathbb{K}+m) \gamma_{\nu}\left[\delta_{\mu 0} \delta_{\nu 0} \rho_{L}\left(q_{0}, q\right)+\mathcal{P}_{\mu \nu}^{T} \rho_{T}\left(q_{0}, q\right)\right] .
\end{aligned}
$$

In Eq. (17), $f$ and $\widetilde{f}$ are Bose-Einstein and Fermi-Dirac distribution functions $(\beta=1 / T)$

$$
f\left(q_{0}\right)=\frac{1}{\mathrm{e}^{\beta q_{0}}-1}, \quad \widetilde{f}\left(k_{0}-\mu\right)=\frac{1}{\mathrm{e}^{\beta\left(k_{0}-\mu\right)}+1} .
$$

The damping rates for the quasiparticles and antiquasiparticles are thus obtained after multiplying Eq. (17) by the corresponding projectors and taking a Dirac trace, evaluating the final expression on the particles mass shell ${ }^{1}$

$$
\gamma_{ \pm}=\left.\mp \operatorname{Im} \Sigma_{ \pm}\left(p_{0}+i \eta, \mathbf{p}\right)\right|_{p_{0} \text { on-shell }} .
$$

To obtain the damping rate of a quasiparticle one has to evaluate the imaginary part of its self-energy on the pole of the dressed propagator. However, up to corrections of order $e^{2}$, it would be enough to consider the above expressions at $p_{0}= \pm E$, as the corrections introduced by $\Sigma_{ \pm}$only displace these poles by an amount proportional to $e^{\overline{2}}$. In this case, after evaluating the Dirac traces we find, with $\mathbf{k}=\mathbf{p}-\mathbf{q}$,

$$
\begin{aligned}
\gamma_{ \pm}= & \pm \frac{\pi e^{2}}{E} \int \frac{d^{3} q}{(2 \pi)^{3}} \int_{-\infty}^{\infty} \frac{d k_{0}}{2 \pi} \rho_{f}\left(k_{0}\right) \int_{-\infty}^{\infty} \frac{d q_{0}}{2 \pi} \\
& \times\left[1+f\left(q_{0}\right)-\tilde{f}\left(k_{0}-\mu\right)\right] \delta\left(p_{0}-k_{0}-q_{0}\right) \\
& \times\left\{\left[p_{0} k_{0}+\mathbf{p} \cdot \mathbf{k}+m^{2}\right] \rho_{L}\left(q_{0}, q\right)\right. \\
& \left.+2\left[p_{0} k_{0}-(\mathbf{p} \cdot \hat{\mathbf{q}})(\mathbf{k} \cdot \hat{\mathbf{q}})-m^{2}\right] \rho_{T}\left(q_{0}, q\right)\right\}\left.\right|_{p_{0}= \pm E} .
\end{aligned}
$$

Equation (20) gives the general expression for the damping rates for any value of $T, \mu$ and $m$. At very high temperature, these damping rates are infrared (IR) logarithmic divergent, even after including the screening corrections. This is due to the soft photon contribution, as $f\left(q_{0}\right) \sim T / q_{0}$ for $q_{0}$ $\ll T$. Perturbation theory fails to provide the damping rates at high $T[12]$. A nonperturbative treatment to resum the leading order divergences was proposed in [13] to find a nonexponential decay law for the quasiparticles.

In this paper we are concerned with the ultradegenerate limit, when $T=0$. In this case several simplifications occur. The damping rates are IR finite after the inclusion of the screening effects [9], as opposed to what happens at high $T$. For $T=0,\left[1+f\left(q_{0}\right)\right]=\Theta\left(q_{0}\right)$, where $\Theta$ is the step function. For $T=0$ the fermion distribution function is $\widetilde{f}\left(E_{k}\right.$ $-\mu)=\Theta\left(\mu-E_{k}\right)$, while $\widetilde{f}\left(-E_{k}-\mu\right)=1-\widetilde{f}\left(E_{k}+\mu\right)=1$.

From this point on, it is convenient to treat separately the electron and positron damping rates, as different phase-space restrictions arise in the two cases. If we concentrate in the soft photon region, we can approximate

$$
E_{k}=\sqrt{|\mathbf{p}-\mathbf{q}|^{2}+m^{2}} \simeq E-\mathbf{v} \cdot \mathbf{q},
$$

\footnotetext{
${ }^{1}$ At this point, one can check that the damping rate of the quasiparticles does not depend on their helicities, by using the helicity projectors of Eq. (8).
} 
where $\mathbf{v}=\mathbf{p} / E$ is the velocity of the fermion. We thus find

$$
\begin{aligned}
\gamma_{+} \simeq & \frac{e^{2}}{8 \pi^{2} v} \int_{q \text { soft }} q d q d q_{0}\left[\left(\Theta\left(q_{0}\right)-\Theta\left(\mu-E+q_{0}\right)\right)\right. \\
& \left.\times\left\{\rho_{L}\left(q_{0}, q\right)+v^{2}\left(1-\cos ^{2} \theta\right) \rho_{T}\left(q_{0}, q\right)\right\}\right], \\
\gamma_{-} \simeq & -\frac{e^{2}}{8 \pi^{2} v} \int_{q \text { soft }} q d q d q_{0}\left[\Theta ( - q _ { 0 } ) \left\{\rho_{L}\left(-q_{0}, q\right)\right.\right. \\
& \left.\left.+v^{2}\left(1-\cos ^{2} \theta\right) \rho_{T}\left(-q_{0}, q\right)\right\}\right],
\end{aligned}
$$

where $q_{0}=q v \cos \theta$.

The damping rates for the electron and the positron thus only differ in the phase-space restrictions of these two types of particles. One can interpret the above equations as follows. A particle or antiparticle, with energy $\pm E$ is scattered to a state of energy $\pm E_{k}$, respectively, creating a particlehole pair. For the electron, $E_{k}$ is forced to be above the Fermi energy, because of Pauli blocking. This last restriction is absent in the case of the positron.

For a quasiparticle with velocity close to the Fermi velocity, we can further approximate $v \approx v_{F}$ in Eq. (22a). From the fact that the spectral functions $\rho_{L, T}$ in Eqs. (22) are evaluated for values of $q_{0}^{2} \leqslant q^{2} v_{F}^{2}$, we see that it is only the part of the spectral function corresponding to Landau damping [the functions $\beta_{L, T}$ in Eq. (A7)], that contributes to the integrals. Using the explicit values of spectral densities as given in Appendix A, one can evaluate the above integrals numerically. Analytical expressions can be obtained for the interesting case $|E-\mu| \ll M$ (so this includes the case of quasiholes). In this regime we find at leading order

$$
\gamma_{+} \sim \frac{e^{2}}{24 \pi}|E-\mu|+\frac{e^{2}}{64 v_{F}^{2} M}(E-\mu)^{2}+\mathcal{O}\left(|E-\mu|^{3}\right),
$$

which generalizes the expressions obtained in Refs. $[9,11]$ for the case $v_{F} \neq 1$. In the above equations $M$ $=\sqrt{e^{2} \mu^{2} v_{F} / \pi^{2}}$ is the Debye mass. The first terms in the right-hand side of Eqs. (23) are due to scattering processes with exchange of soft magnetic photons, while the second is due to the exchange of soft electric ones. As can be seen the magnetic contribution is suppressed with respect to the electric one by a factor $v_{F}^{2}$. Therefore, the electric contribution is dominant for $v_{F} \ll 1$. In the ultrarelativistic limit, $v_{F}=1$, the damping rate of the electron is dominated by the magnetic contribution.

The damping rate of a quasiparticle, or a quasihole, which lives close to the Fermi surface can then be expressed as a power series in $|E-\mu|$. If this parameter is large, then $\gamma_{+}$is large, and the lifetime of these excitations is so short, that it does not make sense to talk about quasiparticles or quasiholes. On the contrary, when the fermion energy approaches the Fermi energy, its lifetime tends to infinity. In particular, from Eq. (23) one deduces that the Fermi sea is stable. We should point out also the very different contribution to the energy dependence of $\gamma_{+}$from the electric and magnetic interactions. The quadratic dependence on $(E-\mu)$ of $\gamma_{+}$can be entirely understood as arising from the short-ranged character of the electric interactions in the plasma, and also the phase-space restrictions of electron-electron scattering (see Appendix B). Magnetic interactions are not short ranged, but only suffer a weak dynamical screening due to Landau damping. The linear dependence on $(E-\mu)$ is also a product of Landau damping and phase-space restrictions.

We now consider the damping rate of the antiquasiparticle. Let us first stress that for a positron pair annihilation also contributes to its damping rate. However, this is a process that occurs at order $e^{4}$, and it can be computed by taking the imaginary part of a two-loop correction to the fermion self-energy. In a weak-coupling expansion, the damping rate of the positron is dominated by the scattering of the positron with the electrons of the Fermi sea. For a positron with velocity $v \approx v_{F}$, we can evaluate numerically Eq. (22b). The only difference with respect to the computation of $\gamma_{+}$comes from the different phase-space restrictions for antifermions, or in other words, the different domain of integration of the integrals. We find at leading order

$$
\gamma_{-} \sim e^{2} M\left(\frac{v_{F}^{2}}{24 \pi}+\frac{1}{64}\right)
$$

which agrees for $v_{F}=1$ with the result of Ref. [11].

The on-shell real part of the self-energy can be obtained from the general expression (16), just by using a principal value prescription to evaluate the integral after the analytical continuation to Minkowski space is done. However, it is much simpler to reconstruct it from the value of the on-shell imaginary part, using a Kramers-Kroning dispersion relation, which gives the value of the real part, up to a constant.

If $f_{ \pm}(\omega)$ is an analytic function in the upper or lower complex plane, respectively, then from the Cauchy theorem, its real part is given as a function of its imaginary part as

$$
\operatorname{Re} f_{ \pm}(\omega)= \pm \frac{P P}{\pi} \int_{-\infty}^{\infty} \frac{d \omega^{\prime}}{\omega^{\prime}-\omega} \operatorname{Im} f_{ \pm}\left(\omega^{\prime}\right)+C_{\infty}
$$

where $P P$ denotes the principal value of the integral along the real axis, and $C_{\infty}$ is a subtraction constant needed in case that $f_{ \pm}$does not vanish for $|\omega| \rightarrow \infty$.

Since we have computed the damping rate $\gamma_{+}$for values $|E-\mu| \ll M$, the only energy domain where the concept of quasiparticle makes sense, we will use the dispersion relation using a cutoff which implements this constraint, that is with cutoffs $\Lambda_{ \pm}=\mu \pm M$. We thus find

$$
\begin{aligned}
\operatorname{Re} \Sigma_{+}(E, p) \sim & \operatorname{Re} \Sigma_{+}(\mu, p)+\frac{e^{2}}{12 \pi^{2}}(E-\mu) \ln \frac{M}{|E-\mu|} \\
& +\frac{e^{2}}{32 \pi v_{F}^{2}}|E-\mu|+\mathcal{O}\left((E-\mu)^{2}\right) .
\end{aligned}
$$


The value of the energy-independent constant $\operatorname{Re} \Sigma_{+}(E$ $=\mu, p)$, which renormalizes the chemical potential, can only be determined from the explicit evaluation of Eq. (16).

We now use the Kramers-Kroning dispersion relation for the antifermions, also imposing a cutoff in the dispersion relation that guarantees that the momentum of the particle is not far away from the Fermi momentum. We thus find

$$
\begin{aligned}
\operatorname{Re} \Sigma_{-}(-E, p) \sim & \operatorname{Re} \Sigma_{-}(-\mu, p)+\left(\frac{e^{2} v_{F}^{2}}{12 \pi^{2}}+\frac{e^{2}}{32 \pi}\right)(\mu-E) \\
& +\mathcal{O}\left((\mu-E)^{2}\right)
\end{aligned}
$$

The leading logarithmic behavior of the real part of the one-loop self-energy of a quark in the high baryonic limit of QCD has been obtained in the ultrarelativistic limit in Ref. [15]. There the same leading logarithmic dependence in the energy as in Eq. (26) has been found. We should stress here that this can only be valid for the quark excitations, but not for the antiquarks ones.

\section{B. Nonrelativistic limit}

In this subsection we take the nonrelativistic (nr) limit of the expressions computed previously, restoring the fundamental constant $c$ in the equations. The $\mathrm{nr}$ limit corresponds to $v_{F} \ll c$. The antiparticles then decouple. In such a case, the contribution from the magnetic sector to the fermion selfenergy is suppressed by a factor $\left(v_{F} / c\right)^{2}$ with respect to the electric sector one. The electric effects are thus dominant.

The lifetime of an electron $\tau$ is defined as $1 / 2 \gamma_{+}$. We neglect the magnetic contribution to the damping rate, and express the electric contribution in terms of the plasma frequency $\omega_{p}^{2}=\frac{1}{3} M^{2} v_{F}^{2}$

$$
\frac{1}{\tau}=\frac{\sqrt{3} \pi^{2} \omega_{p}}{32}\left(\frac{E-\mu}{\mu}\right)^{2} \frac{c^{4}}{v_{F}^{4}} .
$$

The relativistic and nonrelativistic chemical potentials differ by the rest mass of the particle, $\mu^{2}=\mu_{n r}^{2}+m^{2} c^{4}$. For $p^{2} \ll m^{2} c^{2}, E=m c^{2}+\epsilon_{n r}+\mathcal{O}\left(p^{4} / m^{4} c^{2}\right)$, where $\epsilon_{n r}=p^{2} / 2 m$. Therefore,

$$
\frac{1}{\tau} \rightarrow \frac{\sqrt{3} \pi^{2} \omega_{p}}{32} \frac{\left(\epsilon_{n r}-\mu_{n r}\right)^{2}}{m^{2} c^{4}} \frac{c^{4}}{v_{F}^{4}}=\frac{\sqrt{3} \pi^{2} \omega_{p}}{128}\left(\frac{\epsilon_{n r}-\mu_{n r}}{\mu_{n r}}\right)^{2},
$$

where in the last equality we have used $\mu_{n r}=\epsilon_{F}=\frac{1}{2} m v_{F}^{2}$. The above expression agrees with the computation of the lifetime of an electron in a nonrelativistic quantum liquid, using the random-phase approximation [see Eq. (5.134c) of [4]].

Since in the nr limit the difference $(E-\mu) \rightarrow\left(\epsilon_{n r}-\mu_{n r}\right)$ $+\mathcal{O}\left(p^{4} / m^{4} c^{2}\right)$, we also reproduce the dispersion relations due to magnetic interactions of nonrelativistic electrons computed in [5].

\section{CONCLUSIONS}

We have derived the one-loop dispersion relations of quasiparticles and antiquasiparticles with momentum close to the Fermi momentum in a relativistic electromagnetic plasma, recovering in the nonrelativistic limit the results of Refs. [4,5]. As already emphasized in those papers, the longranged character of the magnetic interactions spoils the normal Fermi-liquid behavior of the plasma. This effect is fully dominant when the Fermi velocity $v_{F}$ is close to the velocity of light. We have also found that the medium modifies in a different way the propagation properties of particles and antiparticles. This can be simply understood from their different phase-space restrictions when they scatter with the electrons of the Fermi sea. We should also emphasize that our results are gauge independent. This is because we have computed the one-loop self-energy on mass-shell. Off-shell, the one-loop self-energy (16) is a gauge-dependent function.

While we have concentrated our study to QED plasmas, our results can be easily transported to QCD, only by replacing the electromagnetic coupling constant by the QCD one and taking into account some additional color factors. In the superconducting phase of $\mathrm{QCD}$, the dispersion relations of quarks and antiquarks would be modified in a different way according to whether or not these form Cooper pairs. The dispersion relation for antiquarks in the presence of a color gap has not yet been determined, while it is still necessary to understand how antiquarks propagate in a color superconducting medium.

\section{ACKNOWLEDGMENTS}

I want to thank M. Le Bellac, with whom this work was initiated, and M. Thoma and M. Tytgat for useful discussions.

\section{APPENDIX A: SPECTRAL FUNCTIONS FOR HDL PHOTON PROPAGATORS}

The spectral functions $\rho_{L, T}$ for the resummed propagators $\Delta_{L, T}$ can be found in [1] for the case of an ultrarelativistic $(m=0)$ plasma. In the case of a ultradegenerate plasma, they can also be determined when $m \neq 0$ [8]. For completeness, we will present them below. In this case, the Debye mass is $M^{2}=e^{2} \mu^{2} v_{F} / \pi^{2}$, where $v_{F}$ is the Fermi velocity, defined as the ratio between the Fermi momentum and the Fermi energy, $v_{F}=p_{F} / \mu$. The spectral functions of the resummed HDL propagators are computed from their imaginary part

$$
\rho_{L, T}\left(q_{0}, \mathbf{q}\right)=2 \operatorname{Im} \Delta_{L, T}\left(q_{0}+i \epsilon, \mathbf{q}\right) .
$$

These functions can be written in terms of a contribution of the poles of the propagators, plus another one arising from Landau damping:

$$
\begin{aligned}
\frac{\rho_{L, T}\left(q_{0}, q\right)}{2 \pi}= & Z_{L, T}\left[\delta\left(q_{0}-\omega_{L, T}(q)\right)-\delta\left(q_{0}+\omega_{L, T}(q)\right)\right] \\
& +\beta_{L, T}\left(q_{0}, q\right) .
\end{aligned}
$$

The poles $\omega_{L, T}$ are solutions of the dispersion relations 


$$
\begin{aligned}
& \omega_{L}^{2}(q)= \omega_{p}^{2} \frac{3 \omega_{T}^{2}(q)}{v_{F}^{2} q^{2}}\left[\frac{\omega_{T}(q)}{2 v_{F} q} \ln \frac{\omega_{T}(q)+v_{F} q}{\omega_{T}(q)-v_{F} q}-1\right], \\
& 0 \leqslant q<q_{\max }, \\
& \omega_{T}^{2}(q)=q^{2}+\omega_{p}^{2} \frac{3 \omega_{T}^{2}(q)}{2 v_{F}^{2} q^{2}}\left[1+\frac{1}{2}\left(\frac{v_{F} q}{\omega_{T}(q)}-\frac{\omega_{T}(q)}{v_{F} q}\right)\right. \\
&\left.\times \ln \frac{\omega_{T}(q)+v_{F} q}{\omega_{T}(q)-v_{F} q}\right], \\
& 0 \leqslant q<\infty,
\end{aligned}
$$

where $\omega_{p}^{2}=\frac{1}{3} M^{2} v_{F}^{2}$ is the plasma frequency, and

$$
q_{\max }=\left(\frac{1}{2 v_{F}} \ln \frac{1+v_{F}}{1-v_{F}}-1\right)^{1 / 2} M,
$$

in the maximum momentum at which the plasmon can propagate. The above dispersion relations have to be solved numerically. It is possible to obtain analytically the small $q$ behavior of their solutions. For $q \ll \omega_{p}$
The functions $Z_{L, T}$ are the residues of $\Delta_{L, T}$ evaluated at their poles, and are given by

$$
Z_{L}(q)=\frac{\omega_{L}\left(\omega_{L}^{2}-v_{F}^{2} q^{2}\right)}{q^{2}\left(3 \omega_{p}^{2}-\left(\omega_{L}^{2}-v_{F}^{2} q^{2}\right)\right)}
$$

$\omega_{T}^{2}(q) \rightarrow \omega_{p}^{2}+q^{2}\left(1+\frac{v_{F}^{2}}{5}\right), \quad \omega_{L}^{2}(q) \rightarrow \omega_{p}^{2}+\frac{3}{5} v_{F}^{2} q^{2}$

$$
Z_{T}(q)=\frac{\omega_{T}\left(\omega_{T}^{2}-v_{F}^{2} q^{2}\right)}{3 \omega_{p}^{2} \omega_{T}^{2}+\left(\omega_{T}^{2}+q^{2}\right)\left(\omega_{T}^{2}-v_{F}^{2} q^{2}\right)-2 \omega_{T}^{2}\left(\omega_{T}^{2}-q^{2}\right)}
$$

The pole contribution to the spectral functions is only nonvanishing above the light cone. The Landau damping pieces of the spectral functions are nonvanishing only for $q_{0}^{2} \leqslant q^{2} v_{F}^{2}$ and are given by

$$
\begin{aligned}
& \beta_{L}\left(q_{0}, q\right)=\frac{M^{2} x \Theta\left(1-x^{2}\right)}{2\left[q^{2}+M^{2}\left(1-\frac{x}{2} \ln \left|\frac{x+1}{x-1}\right|\right)\right]^{2}+\frac{M^{4} \pi^{2} x^{2}}{4}}, \\
& \beta_{T}\left(q_{0}, q\right)=\frac{M^{2} v_{F}^{2} x\left(1-x^{2}\right) \Theta\left(1-x^{2}\right)}{\left[2 q^{2}\left(x^{2} v_{F}^{2}-1\right)-M^{2} x^{2} v_{F}^{2}\left(1+\frac{\left(1-x^{2}\right)}{2 x} \ln \left|\frac{x+1}{x-1}\right|\right)\right]^{2}+\frac{M^{4} v_{F}^{4} \pi^{2} x^{2}\left(1-x^{2}\right)^{2}}{4}},
\end{aligned}
$$

where $x=q_{0} / q v_{F}$.

\section{APPENDIX B: LUTTINGER'S THEOREM}

The dependence on $(E-\mu)^{2}$ of the damping rate of a fermion with energy above $\mu$ can be understood completely as arising from phase-space restrictions of fermion-fermion scattering, in the case where the interactions are short-ranged and repulsive. The argument, due to Luttinger [14], is simple. We present it below. Let us consider the decay rate of a fermion with energy $E$ which interacts with a fermion with energy $E_{k}$ inside the Fermi sea. As a result, two new particles appear, with energies $E_{k^{\prime}}$ and $E_{p^{\prime}}$, respectively, which are outside the Fermi sea. The total decay rate is then given by

$$
\Gamma(E)=\frac{1}{E} \int \frac{d^{3} p^{\prime}}{(2 \pi)^{3}} \frac{\left(1-\Theta\left(\mu-E_{p^{\prime}}\right)\right)}{2 E_{p^{\prime}}} \int \frac{d^{3} k}{(2 \pi)^{3}} \frac{\Theta\left(\mu-E_{k}\right)}{2 E_{k}} \int \frac{d^{3} k^{\prime}}{(2 \pi)^{3}} \frac{\left(1-\Theta\left(\mu-E_{k^{\prime}}\right)\right)}{2 E_{k^{\prime}}}(2 \pi)^{4} \delta^{(4)}\left(P+K-P^{\prime}-K^{\prime}\right)|\mathcal{M}|^{2},
$$

where $|\mathcal{M}|^{2}$ is the scattering matrix element squared. After performing the $p^{\prime}$ integral

$$
\Gamma(E)=\frac{2 \pi}{E} \int \frac{d^{3} k}{(2 \pi)^{3}} \frac{\Theta\left(\mu-E_{k}\right)}{2 E_{k}} \int \frac{d^{3} k^{\prime}}{(2 \pi)^{3}} \frac{\left[1-\Theta\left(\mu-E_{k^{\prime}}\right)\right]}{2 E_{k^{\prime}}} \frac{\left[1-\Theta\left(\mu-E_{\mathbf{p}+\mathbf{k}-\mathbf{k}^{\prime}}\right)\right]}{2 E_{\mathbf{p}+\mathbf{k}-\mathbf{k}^{\prime}}} \delta\left(E+E_{k}-E_{\mathbf{p}+\mathbf{k}-\mathbf{k}^{\prime}}-E_{k^{\prime}}\right)|\mathcal{M}|^{2} .
$$


We now make the change of variables

$$
E_{k}=\mu-t_{k}, \quad E_{k^{\prime}}=\mu+t_{k^{\prime}}, \quad E_{\mathbf{p}+\mathbf{k}-\mathbf{k}^{\prime}}=\mu+t_{\mathbf{p}+\mathbf{k}-\mathbf{k}^{\prime}},
$$

where the $t_{i}$ variables are positive quantities. The delta function of energy conservation imposes

$$
E-\mu=t_{k}+t_{k^{\prime}}+t_{\mathbf{p}+\mathbf{k}-\mathbf{k}^{\prime}},
$$

which is only valid for $E-\mu \geqslant 0$. The maximum value that each one of the variables $t_{i}$ can achieve is $E-\mu$, while the minimum is zero. Using the energies of the particles as integration variables, we see that the integration is always performed over an energy shell of thickness $E-\mu$. If $E-\mu$ $\ll \mu$, then the values of the energy variables inside the integral can be substituted by the Fermi energy. One then finally reaches

$$
\Gamma(E) \propto \int_{\mu-(E-\mu)}^{\mu} d E_{k} \int_{\mu}^{\mu+(E-\mu)} d E_{k^{\prime}}
$$

and thus $\Gamma(E) \propto(E-\mu)^{2}$. A similar argument can be applied for a quasihole to get the energy dependence of its damping rate.

In the case we studied in this paper, the electric interactions can be considered as short ranged, because of Debye screening; they thus give a contribution to the damping rate of electrons as expected from Luttinger's theorem. The above arguments fail in the case of magnetic interactions, as those are not short ranged, but rather suffer a weak dynamical screening, where the energies themselves play the role of infrared cutoffs in the above integrals.
[1] M. Le Bellac, Thermal Field Theory (Cambridge University Press, Cambridge, England, 1996).

[2] F. Wilczek, "QCD in extreme conditions,' hep-ph/0003183.

[3] D. H. Rischke and R. D. Pisarski, "'Color superconductivity in cold, dense quark matter,' nucl-th/0004016.

[4] D. Pines and P. Nozieres, The Theory of Quantum Liquids. Normal Fermi Liquids (Addison-Wesley, Redwood City, 1988), Vol. 1.

[5] T. Holstein, R. Norton, and P. Pincus, Phys. Rev. B 8, 264 (1973); M. Yu. Reizer, ibid. 40, 11571 (1989); S. Chakravarty, R. E. Norton, and O. F. Syljuasen, Phys. Rev. Lett. 74, 1423 (1995).

[6] M. Alford, K. Rajagopal, and F. Wilczek, Nucl. Phys. B537, 443 (1999).
[7] R. D. Pisarski, Phys. Rev. Lett. 63, 1129 (1989); E. Braaten and R. D. Pisarski, Nucl. Phys. B337, 569 (1990).

[8] C. Manuel, Phys. Rev. D 53, 5866 (1996).

[9] M. Le Bellac and C. Manuel, Phys. Rev. D 55, 3215 (1997).

[10] R. D. Pisarski, Physica A 158, 146 (1989).

[11] B. Vanderheyden and J. Ollitrault, Phys. Rev. D 56, 5108 (1997).

[12] R. D. Pisarski, Phys. Rev. D 47, 5589 (1993).

[13] J. Blaizot and E. Iancu, Phys. Rev. Lett. 76, 3080 (1996); Phys. Rev. D 55, 973 (1997).

[14] J. M. Luttinger, Phys. Rev. 121, 942 (1961).

[15] W. E. Brown, J. T. Liu, and H. Ren, Phys. Rev. D 62, 054013 (2000). 